\title{
Left ventricular fractional shortening as a novel predictor of clinical outcomes in patients with coronary artery disease after undergoing PCI: A Retrospective Cohort Study
}

Qian-Qian Guo

Zhengzhou University First Affiliated Hospital

Jun-Nan Tang

Zhengzhou University First Affiliated Hospital

Xu-Ming Yang

Henan University of Science and Technology Affiliated First Hospital

Jian-Chao Zhang

Zhengzhou University First Affiliated Hospital

Meng-Die Cheng

Zhengzhou University First Affiliated Hospital

Feng-Hua Song

Zhengzhou University First Affiliated Hospital

Zhi-Yu Liu

Zhengzhou University First Affiliated Hospital

Kai Wang

Zhengzhou University First Affiliated Hospital

Li-Zhu Jiang

Zhengzhou University First Affiliated Hospital

Lei Fan

Zhengzhou University First Affiliated Hospital

Xiao-Ting Yue

Zhengzhou University First Affiliated Hospital

Yan Bai

Zhengzhou University First Affiliated Hospital

Xin-Ya Dai

Zhengzhou University First Affiliated Hospital

Zeng-Lei Zhang

Zhengzhou University First Affiliated Hospital

Ru-Jie Zheng

Zhengzhou University First Affiliated Hospital

Ying-Ying Zheng ( $\square$ zhengying527@163.com )

Jin-Ying Zhang

Zhengzhou University First Affiliated Hospital

\section{Research}

Keywords: left ventricular fractional shortening, coronary artery disease, percutaneous coronary intervention, clinical outcomes

Posted Date: August 2nd, 2020

DOI: https://doi.org/10.21203/rs.3.rs-49052/v1

License: @ (1) This work is licensed under a Creative Commons Attribution 4.0 International License. Read Full License 


\section{Abstract}

Background: Even though great advances have been made in the treatment of coronary artery disease (CAD) owing to coronary revascularization and modern antiremodeling therapy, it remains the major cause of cardiac morbidity and mortality worldwide. Risk stratification in CAD patients is primarily based on left ventricular ejection fraction (LVEF), risk scores, and some serum markers. The value of baseline Left ventricular fractional shortening (LVFS) level in predicting the clinical outcomes has not yet been determined.

Methods: In this retrospective cohort study, a total of 3561 patients were enrolled in Clinical Outcomes and Risk Factors of Patients with CAD after percutaneous coronary intervention (PCl), from January 2013 to December 2017. After excluding patients without echocardiography data, we finally enrolled 2787 patients. These patients were divided into two groups according to LVFS value. The lower group (LVFS $<31 \%$, $n=741$ ), the higher group (LVFS $\geq 31 \%, n=2046$ ). The average follow-up time were $37.59 \pm 22.24$ months.

Results: We found that there were significant differences between the two groups in the incidence of all-cause mortality $(A C M)(P<0.001)$, cardiac mortality (CM) $(P<0.001)$, major adverse cardiovascular events (MACEs) $(P<0.05)$ and major adverse cardiovascular and cerebrovascular events (MACCEs) $(P<0.05)$. Multivariate Cox regression analyses showed that LVFS was an independent predictor for ACM (hazard ratio [HR]:0.473 [95\% confidence interval [Cl]:0.290-0.772], $P=0.003), \mathrm{CM}$ (HR: 0.393 [95\% Cl:0.213-0.725], $P=0.003$ ) in acute coronary syndrome (ACS) patients but that it was an independent predictor for only the incidence of CM (HR: 0.153 [95\% Cl:0.046-0.504],P=0.002) in stable CAD patients.

Conclusion This study indicates that baseline LVFS is an independent and novel predictor of adverse long-term outcomes in CAD patients who underwent $\mathrm{PCl}$.

\section{Background}

Coronary artery disease (CAD) is currently the leading cause of human death around the world[1]. Percutaneous coronary intervention (PCl) has been known to be effective in the management of CAD[2]. With the improvement of operators' experience and techniques, $\mathrm{PCI}$ has been widely used in the revascularization recently. However, adverse clinical events remain an important issue in short-and long-term outcomes after successful PCI[3], thus identifying factors that can predict the prognosis of patients with CAD after successful PCI is warrant for therapeutic prevention.

Left ventricular fractional shortening(LVFS) is a index of echocardiography, which is used to evaluate the function and structure of the heart, the measurement indexes of conventional echocardiography include interventricular septal thickness, LV end-diastolic dimension (LVEDD, $\mathrm{mm}$ ), LV end-systolic dimension (LVESD, mm), and left atrial size, left ventricular ejection fraction(LVEF) and so on[4]. Among them LVFS can be used to evaluate the left ventricular systolic function, and it is calculated according to the formula: LVFS $(\%)=100 \times[($ LVEDD LVESD)/LVEDD][5]. LVEF is the central measure of left ventricular systolic function[6] and can be used to predict the clinical outcomes in patients with congestive heart failure, after myocardial infarction, and after revascularization[7-9]. However, there is few studies focusing on the the relationship between LVFS and adverse outcome in patients with CAD. Therefore, our study examined the LVFS index of patients with CAD undergoing PCl and discussed the effect of LVFS index on long-term clinical outcomes.

\section{Methods}

\section{Study design and population}

3561 CAD patients hospitalized and received at least one stent via implantation in the First Affiliated Hospital of Zhengzhou University from 2013 to 2017 were recruited in the Clinical Outcomes and Risk Factors of Patients with Coronary Heart Disease after PCl (CORFCHD-PCl) study. It is a large, single-center retrospective cohort study. Patients with dysfunction of the liver, serious heart failure, congenital disease, serious dysfunction of the kidney are excluded. This study protocol complies with the declaration of Helsinki, also the ethics committee of the First Affiliated Hospital of Zhengzhou University has approved the study and waived the need of obtaining informed consent from eligible patients, as this is a retrospective study.

In this study, 3561 patients were initially evaluated. 774 patients were excluded for insufficient echocardiography data. Finally, 2787 patients were admitted including 2107 ACS patients and 680 stable CAD patients.(Figure 1).

\section{Clinical and Demographic Characteristics Collection}

We collected and recorded clinical data, demographic data, cardiovascular risk factors, imaging and laboratory data including age, sex, history of hypertension, history of diabetes mellitus, smoking and drinking status, fasting blood glucose, blood urea nitrogen, creatinine, Uric 
Acid, lipid parameters, angiographic results and echocardiography. During the follow-up period, we further recorded the use of drugs in these patients.

\section{Echocardiography}

Two-dimensional echocardiography was performed according to the American Society of Echocardiography standards, by a cardiology resident. The selected parameters included LVEF and LVFS using the volume method and visual estimation, LVEDD, LVESD, interventricular septal thickness at end diastole (IVSd), and the left ventricular posterior wall thickness at end diastole (LVPWd) were also measured.

\section{Definitions}

Hypertension was defined as a systolic blood pressure of $\geq 140 \mathrm{mmHg}$ and/or a diastolic blood pressure of $\geq 90 \mathrm{mmHg}$ on at least three resting measurements on at least two separate health care visits according to the American Heart Association recommendations or with the use of any antihypertensive drug [10]. Diabetes mellitus was defined as fasting blood glucose $\geq 7.1 \mathrm{mmol} / \mathrm{L}$ or 2-hour post-load glucose $\geq 11.1 \mathrm{mmol} / \mathrm{L}$ or current use of anti-diabetic medications[11]. Smoking and drinking status was defined as current tobacco and alcohol use.

\section{Endpoints}

The primary endpoint is long-term mortality, including all-cause mortality (ACM) and cardiac mortality (CM)[12]. And the secondary endpoints include major adverse cardiac events (MACE) (a composite of cardiac death, recurrent myocardial infarction and target vessel reconstruction), major adverse cardiocerebrovascular events (MACCE) defined as MACEs plus stroke[13] and heart failure. Stroke refers to acute neurological impairment caused by cerebrovascular diseases, including hemorrhage, embolism, thrombosis or aneurysm rupture, lasting for more than 24 hours[13]. All events were assessed by an adjudication committee which was blinded to the group of patients.

\section{Follow-up}

In this study, we checked all the medical records and contacted patients or their families by telephone. Patients were followed up at least 2 years. Drug compliance and adverse events were carefully evaluated at each contact.

\section{Statistical Analyses}

All analyses were conducted using the SPSS 23.0 (SPSS, Chicago, Illinois). Continuous data and categorical data were shown as the mean \pm standard deviation and the percentages and frequencies, respectively. The LVFS index was analyzed as a categorical variable divided into two groups on the basis of LVFS cut-off value of $31 \%$. The cut-off value (31\%) is according to the analysis of the ROC curve for the baseline data of the study population. The differences between normally distributed numerical variables were analyzed by a t-test, and non-normally distributed variables were analyzed by the Mann-Whitney U-test or Kruskal-Wallis variance analysis as appropriate. Chi-square tests were used to compare categorical variables. Kaplan-Meier analysis was used to analyze cumulative incidence rate of adverse events. The logrank test was employed for comparing between two groups. Multivariable regression analysis was used to evaluate the predictive value of the LVFS index for outcomes during follow-up. Hazard ratios (HRs) and 95囚 confidence intervals (Cls) were calculated, and a two-sided P value $<0.05$ was considered statistically significant.

\section{Results}

\section{ROC analysis for LVEF and LVFS predicting adverse outcomes in patients with CAD after undergoing PCl}

In ROC analyses, baseline LVEF and LVFS predicted adverse outcomes in patients with CAD after undergoing PCI [area under the curve (AUC): 0.618; $P<0.001$ and AUC: 0.655; $P<0.001$, respectively], as shown in Fig. 2.

\section{Baseline Characteristics}

Patients finally included in the study was categorized into two groups according to the LVFS value: the low LVFS group $(<31 \%, \mathrm{n}=741)$, the high LVFS group $(\geq 31 \%, n=2046)$. As shown in Table 1, in total, variables, including age, gender,smoking, diabetes mellitus, LVEF, systolic blood pressure (SBP), diastolic blood pressure (DBP), hypertension, white blood cell (WBC), plate count (PLT), uric acid (UA), fasting blood glucose (FBG), creatinine (Cr), albumin, high-density lipoprotein cholesterol (HDL-C), use of asprin, CCB and statin showed significant difference between the two groups (all $P$-values < 0.05). In the ACS patients, we found that age, gender, diabetes mellitus, LVEF, hypertension, SBP, DBP, WBC, Cr, UA, FBG, albumin, use of asprin and CCB were significantly different between the two groups. In the stable CAD (SCAD) 
patients, we found that gender, diabetes mellitus, LVEF, SBP, PLT, UA, FBG, HDL-C, LDL-C, use of asprin, ticagrelor and statin were significantly different between the two groups. 
Table 1

Baseline characteristics

\begin{tabular}{|c|c|c|c|c|c|c|c|c|c|}
\hline \multirow[t]{2}{*}{ Variables } & \multicolumn{3}{|l|}{ Total } & \multicolumn{3}{|l|}{ ACS } & \multicolumn{3}{|l|}{ SCAD } \\
\hline & $\begin{array}{l}\text { Low } \\
\text { (LVFS < } \\
31 \% ; \\
n=741 \text { ) }\end{array}$ & $\begin{array}{l}\text { High (LVFS } \\
\geq 31 \% ; \\
n=2046 \text { ) }\end{array}$ & $P$ & $\begin{array}{l}\text { Low } \\
\text { (LVFS < } \\
31 \% ; \\
n=581)\end{array}$ & $\begin{array}{l}\text { High (LVFS } \\
\geq 31 \% ; \\
n=1526)\end{array}$ & $P$ & $\begin{array}{l}\text { Low } \\
\text { (LVFS< } \\
31 \% ; \\
n=160)\end{array}$ & $\begin{array}{l}\text { High } \\
\text { (LVFS } \geq \\
31 \% ; \\
n=520 \text { ) }\end{array}$ & $P$ \\
\hline Age (years) & $\begin{array}{l}64.23 \pm \\
11.29\end{array}$ & $\begin{array}{l}62.85 \pm \\
10.26\end{array}$ & 0.004 & $\begin{array}{l}64.37 \pm \\
11.17\end{array}$ & $62.95 \pm 9.97$ & 0.005 & $\begin{array}{l}63.83 \pm \\
11.68\end{array}$ & $\begin{array}{l}62.56 \pm \\
11.05\end{array}$ & 0.212 \\
\hline $\begin{array}{l}\text { Male, } \\
\mathrm{n}(\%)\end{array}$ & $\begin{array}{l}547 \\
(74.0 \%)\end{array}$ & $\begin{array}{l}1354 \\
(66.2 \%)\end{array}$ & $\hat{0}_{001}$ & $428(73.7 \%)$ & $\begin{array}{l}1009 \\
(66.1 \%)\end{array}$ & 0.001 & $120(75.0 \%)$ & $345(66.3 \%)$ & 0.041 \\
\hline $\begin{array}{l}\text { Smoking, } \\
n(\%)\end{array}$ & $249(33.7 \%)$ & $600(29.3 \%)$ & 0.028 & 194(33.4\%) & $442(29.0 \%)$ & 0.050 & $56(35.0 \%)$ & $158(30.4 \%)$ & 0.285 \\
\hline $\begin{array}{l}\text { Alcohol drinking, } \\
n(\%)\end{array}$ & $\begin{array}{l}128 \\
(17.3 \%)\end{array}$ & $327(16.0 \%)$ & 0.416 & $98(16.9 \%)$ & $243(15.9 \%)$ & 0.597 & $30(18.8 \%)$ & $84(16.2 \%)$ & 0.468 \\
\hline $\begin{array}{l}\text { Diabetes mellitus, } \\
\mathrm{n}(\%)\end{array}$ & $\begin{array}{l}202 \\
(27.3 \%)\end{array}$ & $444(21.7 \%)$ & 0.002 & $158(27.2 \%)$ & $341(22.3 \%)$ & 0.022 & $44(27.5 \%)$ & $103(19.8 \%)$ & 0.048 \\
\hline LVEF (\%) & $\begin{array}{l}52.55 \pm \\
7.98\end{array}$ & $63.39 \pm 3.74$ & ¿.001 & $\begin{array}{l}52.89 \pm \\
7.86\end{array}$ & $63.48 \pm 3.62$ & ¿. 001 & $\begin{array}{l}51.45 \pm \\
8.38\end{array}$ & $\begin{array}{l}63.14 \pm \\
4.06\end{array}$ & $\hat{0} .001$ \\
\hline Hypertension,n(\%) & $383(51.8 \%)$ & $\begin{array}{l}1159 \\
(56.6 \%)\end{array}$ & 0.025 & $300(51.6 \%)$ & $880(57.7 \%)$ & 0.014 & $84(52.5 \%)$ & $279(53.7 \%)$ & 0.856 \\
\hline $\mathrm{SBP}(\mathrm{mmHg})$ & $\begin{array}{l}129.77 \pm \\
17.72\end{array}$ & $\begin{array}{l}134.46 \pm \\
17.60\end{array}$ & $\hat{0.001}$ & $\begin{array}{l}129.31 \pm \\
17.41\end{array}$ & $\begin{array}{l}134.32 \pm \\
17.83\end{array}$ & $\hat{0.001}$ & $\begin{array}{l}131.51 \pm \\
18.65\end{array}$ & $\begin{array}{l}134.85 \pm \\
16.92\end{array}$ & 0.035 \\
\hline $\mathrm{DBP}(\mathrm{mmHg})$ & $\begin{array}{l}78.15 \pm \\
11.14\end{array}$ & $\begin{array}{l}79.90 \pm \\
11.11\end{array}$ & ¿.001 & $\begin{array}{l}77.98 \pm \\
11.16\end{array}$ & $\begin{array}{l}80.08 \pm \\
11.19\end{array}$ & ¿. 001 & $\begin{array}{l}78.78 \pm \\
11.03\end{array}$ & $\begin{array}{l}79.38 \pm \\
10.84\end{array}$ & 0.544 \\
\hline WBC $\left(x 10^{\wedge} 9\right)$ & $7.32 \pm 2.62$ & $6.76 \pm 1.93$ & $\hat{0}_{001}$ & $7.42 \pm 2.74$ & $6.75 \pm 1.93$ & $\dot{0}_{0.001}$ & $6.89 \pm 2.05$ & $6.80 \pm 1.94$ & 0.581 \\
\hline $\mathrm{Hb}(\mathrm{g} / \mathrm{L})$ & $\begin{array}{l}132.18 \pm \\
15.40\end{array}$ & $\begin{array}{l}132.60 \pm \\
14.26\end{array}$ & 0.703 & $\begin{array}{l}131.63 \pm \\
15.72\end{array}$ & $\begin{array}{l}132.43 \pm \\
13.97\end{array}$ & 0.265 & $\begin{array}{l}134.14 \pm \\
13.94\end{array}$ & $\begin{array}{l}133.10 \pm \\
15.09\end{array}$ & 0.446 \\
\hline PLT $\left(x 10^{\wedge} 9\right)$ & $\begin{array}{l}206.92 \pm \\
66.82\end{array}$ & $\begin{array}{l}205.85 \pm \\
58.80\end{array}$ & ¿.001 & $\begin{array}{l}206.69 \pm \\
68.29\end{array}$ & $\begin{array}{l}205.12 \pm \\
57.26\end{array}$ & 0.125 & $\begin{array}{l}196.52 \pm \\
59.76\end{array}$ & $\begin{array}{l}207.98 \pm \\
63.14\end{array}$ & 0.047 \\
\hline $\mathrm{Cr}(\mathrm{mmol} / \mathrm{L})$ & $\begin{array}{l}76.27 \pm \\
32.19\end{array}$ & $\begin{array}{l}70.81 \pm \\
35.68\end{array}$ & $\hat{0.001}$ & $\begin{array}{l}76.22 \pm \\
31.02\end{array}$ & $\begin{array}{l}70.40 \pm \\
23.95\end{array}$ & $\hat{0.001}$ & $\begin{array}{l}76.34 \pm \\
36.15\end{array}$ & $\begin{array}{l}71.99 \pm \\
57.72\end{array}$ & 0.375 \\
\hline $\mathrm{UA}(\mathrm{mmol} / \mathrm{L})$ & $\begin{array}{l}307.88 \pm \\
95.16\end{array}$ & $\begin{array}{l}293.64 \pm \\
81.85\end{array}$ & ¿.001 & $\begin{array}{l}306.18 \pm \\
93.39\end{array}$ & $\begin{array}{l}293.43 \pm \\
81.85\end{array}$ & 0.002 & $\begin{array}{l}314.09 \pm \\
101.10\end{array}$ & $\begin{array}{l}294.26 \pm \\
81.93\end{array}$ & 0.013 \\
\hline $\mathrm{FBG}(\mathrm{mmol} / \mathrm{L})$ & $6.01 \pm 2.69$ & $5.48 \pm 1.91$ & $\hat{0.001}$ & $6.01 \pm 2.75$ & $5.45 \pm 1.90$ & $\hat{0.001}$ & $5.96 \pm 2.48$ & $5.56 \pm 1.93$ & 0.040 \\
\hline Albumin $(\mathrm{g} / \mathrm{L})$ & $\begin{array}{l}40.22 \pm \\
4.56\end{array}$ & $41.13 \pm 4.60$ & ¿.001 & $\begin{array}{l}40.21 \pm \\
4.57\end{array}$ & $41.16 \pm 4.60$ & ¿.001 & $\begin{array}{l}40.28 \pm \\
4.53\end{array}$ & $\begin{array}{l}41.00 \pm \\
4.58\end{array}$ & 0.087 \\
\hline $\mathrm{TG}(\mathrm{mmol} / \mathrm{L})$ & $1.64 \pm 1.15$ & $1.68 \pm 1.14$ & 0.395 & $1.59 \pm 1.01$ & $1.69 \pm 1.15$ & 0.093 & $1.83 \pm 1.57$ & $1.67 \pm 1.11$ & 0.186 \\
\hline $\mathrm{TC}(\mathrm{mmol} / \mathrm{L})$ & $3.88 \pm 1.06$ & $3.91 \pm 1.00$ & 0.615 & $3.91 \pm 1.05$ & $3.89 \pm 1.00$ & 0.702 & $3.80 \pm 1.08$ & $3.96 \pm 1.00$ & 0.086 \\
\hline $\mathrm{HDL}-\mathrm{C}(\mathrm{mmol} / \mathrm{L})$ & $1.01 \pm 0.29$ & $1.05 \pm 0.27$ & 0.003 & $1.01 \pm 0.30$ & $1.04 \pm 0.26$ & 0.069 & $1.00 \pm 0.25$ & $1.07 \pm 0.30$ & 0.008 \\
\hline LDL-C (mmol/L) & $2.37 \pm 0.83$ & $2.40 \pm 0.84$ & 0.439 & $2.40 \pm 0.84$ & $2.38 \pm 0.85$ & 0.630 & $2.56 \pm 0.80$ & $2.46 \pm 0.82$ & 0.009 \\
\hline
\end{tabular}

ACS acute coronary syndrome, SCAD stable coronary artery disease,ACEl angiotensin-converting enzyme inhibitor, ARB angiotensin receptor antagonist, CCB calcium channel blocker, SBP systolic blood pressure, DBP diastolic blood pressure, BUN blood urea nitrogen, Cr creatinine, UA Uric Acid, FBG fasting blood glucose, Hb hemoglobin, WBC white blood count, PLT platelet,LVEF Left ventricular ejection fraction, TG triglyceride, TC total cholesterol, HDL-C high-density lipoprotein cholesterol, LDL-C low-density lipoprotein cholesterol Note: The boldfaced values indicate $\mathrm{P}<0.05$. 


\begin{tabular}{|c|c|c|c|c|c|c|c|c|c|}
\hline \multirow[t]{2}{*}{ Variables } & \multicolumn{3}{|l|}{ Total } & \multicolumn{3}{|l|}{ ACS } & \multicolumn{3}{|l|}{ SCAD } \\
\hline & $\begin{array}{l}\text { Low } \\
\text { (LVFS < } \\
31 \% ; \\
n=741 \text { ) }\end{array}$ & $\begin{array}{l}\text { High (LVFS } \\
\geq 31 \% ; \\
n=2046)\end{array}$ & $P$ & $\begin{array}{l}\text { Low } \\
\text { (LVFS < } \\
31 \% ; \\
n=581 \text { ) }\end{array}$ & $\begin{array}{l}\text { High (LVFS } \\
\geq 31 \% ; \\
n=1526)\end{array}$ & $P$ & $\begin{array}{l}\text { Low } \\
\text { (LVFS < } \\
31 \% ; \\
n=160 \text { ) }\end{array}$ & $\begin{array}{l}\text { High } \\
\text { (LVFS } \geq \\
31 \% ; \\
n=520 \text { ) }\end{array}$ & $P$ \\
\hline CCB, n(\%) & $99(13.4 \%)$ & $399(19.5 \%)$ & <. 001 & 77(13.3\%) & 294(19.3\%) & 0.001 & $22(13.8 \%)$ & $105(20.2 \%)$ & 0.081 \\
\hline $\begin{array}{l}\beta \text {-blocker, } \\
\mathrm{n}(\%)\end{array}$ & \multicolumn{8}{|c|}{$n(\%)$} & 0.319 \\
\hline ACEI or ARB, $n(\%)$ & $231(31.3 \%)$ & $569(27.8 \%)$ & 0.079 & $174(29.9 \%)$ & $397(26.0 \%)$ & 0.071 & $58(36.3 \%)$ & 172(33.1\%) & 0.504 \\
\hline Clopidogrel, n(\%) & $178(24.1 \%)$ & $486(23.8 \%)$ & 0.880 & $142(24.4 \%)$ & $366(24.0 \%)$ & 0.820 & $36(22.5 \%)$ & $120(23.1 \%)$ & 0.915 \\
\hline Statin, $n(\%)$ & $555(75.1 \%)$ & $1660(81.1 \%)$ & 0.001 & $440(75.7 \%)$ & 1233(80.8\%) & 0.011 & 117(73.1\%) & $427(82.1 \%)$ & 0.017 \\
\hline Ticagrelor, n(\%) & $62(8.4 \%)$ & $187(9.1 \%)$ & 0.599 & $51(8.8 \%)$ & $119(7.8 \%)$ & 0.474 & $11(6.9 \%)$ & $68(13.1 \%)$ & 0.034 \\
\hline Asprin, $n(\%)$ & $580(78.5 \%)$ & 1737(84.9\%) & $\hat{0}_{0.001}$ & $456(78.5 \%)$ & $1281(83.9 \%)$ & 0.004 & $126(78.8 \%)$ & $456(87.7 \%)$ & 0.007 \\
\hline $\begin{array}{l}\text { ACS acute coronar } \\
\text { receptor antagonis } \\
\text { creatinine, UA Uric } \\
\text { fraction, TG triglyc } \\
\text { Note: The boldface }\end{array}$ & $\begin{array}{l}\text { ndrome, SC } \\
\text { CB calcium c } \\
\text { d, FBG fastin } \\
\text { e, TC total ch } \\
\text { alues indicat }\end{array}$ & $\begin{array}{l}\text { stable corone } \\
\text { annel blocker, } \\
\text { blood glucose } \\
\text { lesterol, HDL-C } \\
\text { P<0.05. }\end{array}$ & arte & $\begin{array}{l}\text { ease,ACEI } \\
\text { blood pre } \\
\text { obin, WBC } \\
\text { y lipoprote }\end{array}$ & $\begin{array}{l}\text { giotensin-co } \\
\text { Ire, DBP dias } \\
\text { ite blood co } \\
\text { cholesterol, L }\end{array}$ & $\begin{array}{l}\text { ting e } \\
\text { bloo } \\
\text { PLT p } \\
\text { C low }\end{array}$ & $\begin{array}{l}\text { me inhibito } \\
\text { essure, BUl } \\
\text { let,LVEF Le } \\
\text { isity lipopr }\end{array}$ & $\begin{array}{l}\text { RB angiote } \\
\text { lood urea n } \\
\text { entricular } € \\
n \text { cholester }\end{array}$ & $\begin{array}{l}\text { en, } \mathrm{Cr} \\
\text { ion }\end{array}$ \\
\hline
\end{tabular}

\section{Clinical Outcomes}

As Table 2 shows, the incidence of ACM, CM, MACEs and MACCEs were all significantly different between two groups $(6.4 \%$ vs $2.4 \%, 4.7 \%$ vs $2.3 \%, 13.7 \%$ vs $10.8 \%, 18.1 \%$ vs $14.5 \%$, respectively. And all $P$-values $<0.05)$. while we found that there were no significant differences in the incidence of stroke $(5.0 \%$ vs $4.0 \%, P=0.241)$ and heart failure ( $15.8 \%$ vs $15.1 \%, P=0.633)$ between the two groups. Sub-group analysis suggested that there was a significant difference in the incidence of ACM $(6.2 \%$ vs. $2.4 \%, P<0.001)$ and CM $(4.1 \%$ vs. $1.4 \%, P<0.001)$ between the high LVFS group and the low LVFS group for the ACS patients. For the stable CAD patients, we found significant differences in the incidence of $\operatorname{ACM}(6.9 \%$ vs. $2.7 \%, P=0.027)$ and $\mathrm{CM}(6.9 \%$ vs. $1.2 \%, P<0.001)$ between the two groups. 
Table 2

Outcomes comparison between groups

\begin{tabular}{|c|c|c|c|c|c|c|c|c|c|}
\hline \multirow[t]{2}{*}{ Variables } & \multicolumn{3}{|l|}{ Total } & \multicolumn{3}{|l|}{ ACS } & \multicolumn{3}{|l|}{ SCAD } \\
\hline & $\begin{array}{l}\text { Low } \\
\text { (LVFS < } \\
31 \% ; \\
n=741)\end{array}$ & $\begin{array}{l}\text { High (LVFS } \geq \\
31 \% ; \\
n=2046)\end{array}$ & $P$ & $\begin{array}{l}\text { Low } \\
\text { (LVFS < } \\
31 \% ; \\
n=581)\end{array}$ & $\begin{array}{l}\text { High (LVFS } \\
\geq 31 ; \\
\mathbf{n = 1 5 2 6 )}\end{array}$ & $P$ & $\begin{array}{l}\text { Low } \\
\text { (LVFS < } \\
31 \% ; \\
n=160)\end{array}$ & $\begin{array}{l}\text { High (LVFS } \\
\geq 31 \% ; \\
n=520)\end{array}$ & $P$ \\
\hline $\begin{array}{l}\text { All-cause } \\
\text { mortality, } \\
\mathrm{n}(\%)\end{array}$ & $47(6.4 \%)$ & $50(2.4 \%)$ & $\hat{0} .001$ & $36(6.2 \%)$ & $36(2.4 \%)$ & $\hat{0} .001$ & $11(6.9 \%)$ & $14(2.7 \%)$ & 0.027 \\
\hline $\begin{array}{l}\text { Cardiac } \\
\text { mortality, } \\
\mathrm{n}(\%)\end{array}$ & $35(4.7 \%)$ & $27(2.3 \%)$ & $\hat{0} .001$ & $24(4.1 \%)$ & $21(1.4 \%)$ & $\hat{0} .001$ & $11(6.9 \%)$ & $6(1.2 \%)$ & < 0.001 \\
\hline $\begin{array}{l}\text { MACEs, } \\
n(\%)\end{array}$ & 101(13.7\%) & $220(10.8 \%)$ & 0.037 & 74(12.7\%) & 163(10.7\%) & 0.190 & $27(16.9 \%)$ & $57(11.0 \%)$ & 0.054 \\
\hline $\begin{array}{l}\text { MACCEs, } \\
n(\%)\end{array}$ & $\begin{array}{l}134 \\
(18.1 \%)\end{array}$ & $296(14.5 \%)$ & 0.020 & $100(17.2 \%)$ & $219(14.4 \%)$ & 0.103 & $34(21.3 \%)$ & $77(14.8 \%)$ & 0.066 \\
\hline
\end{tabular}

Note: The boldfaced values indicate $\mathrm{P}<0.05$.

In Figure. 3, Figure. 4 and Figure. 5, as Kaplan-Meier survival analysis revealed, lower LVFS level were apt to have an increasing accumulated risk for ACM and CM in CAD patients, ACS patients and SCAD patients .(all Log rank $P$-values $<0.05$ ).

To access the prognostic value of the LVFS, we performed the multivariable analysis after adjusting for the traditional risk factors, including gender, age, hypertension, diabetes mellitus, smoking, alcohol drinking, Cr, UA, triglyceride (TG) and LDL-C. In total ,we found that patients in the high LVFS group are less likely to occur ACM (HR: 0.458 and 95\% Cl: $0.302-0.696, P<0.001)$ and CM (HR: 0.305 and $95 \%$ Cl: $0.179-$ $0.519, P<0.001)$. (Table 3$)$. In the ACS group, after performing an adjustment of confounders, the risks for ACM and CM events were still significantly different (HR: 0.473 and $95 \% \mathrm{Cl}: 0.290-0.772, P=0.003)$ and (HR: 0.393 and $95 \% \mathrm{Cl}: 0.213-0.725, P=0.003)$. In the stable CAD patients, only CM remained significantly different after adjusting for confounders.

Table 3

Multivariable analyses of LVFS and outcomes

\begin{tabular}{|c|c|c|c|c|c|c|c|c|c|}
\hline \multirow[t]{2}{*}{ Outcomes } & \multicolumn{3}{|l|}{ Total } & \multicolumn{3}{|l|}{ ACS } & \multicolumn{3}{|l|}{ SCAD } \\
\hline & $\begin{array}{l}\text { Adjusted } \\
\text { HR }\end{array}$ & $95 \% \mathrm{Cl}$ & $P$ & $\begin{array}{l}\text { Adjusted } \\
\text { HR }\end{array}$ & $95 \% \mathrm{Cl}$ & $P$ & $\begin{array}{l}\text { Adjusted } \\
\text { HR }\end{array}$ & $95 \% \mathrm{Cl}$ & $P$ \\
\hline $\begin{array}{l}\text { All-cause } \\
\text { mortality }\end{array}$ & 0.458 & $\begin{array}{l}0.302- \\
0.696\end{array}$ & <. 001 & 0.473 & $\begin{array}{l}0.290- \\
0.772\end{array}$ & 0.003 & 0.485 & $\begin{array}{l}0.207- \\
1.136\end{array}$ & 0.096 \\
\hline Cardiac mortality & 0.305 & $\begin{array}{l}0.179- \\
0.519\end{array}$ & $\begin{array}{l}<.001 \\
0.001\end{array}$ & 0.393 & $\begin{array}{l}0.213- \\
0.725\end{array}$ & 0.003 & 0.153 & $\begin{array}{l}0.046- \\
0.504\end{array}$ & 0.002 \\
\hline
\end{tabular}

MACEs major adverse cardiac events,MACCEs main adverse cardiovascular and cerebrovascular events

Note: The boldfaced values indicate $\mathrm{P}<0.05$.

\section{Discussion}

In this retrospective cohort study, high LVFS level ( $\geq 31 \%)$ is associated with an decreased risk of ACM and CM events in patients undergoing $\mathrm{PCl}$. The association remains significant after adjustments for confounders (ACM, HR: 0.458 and 95\% Cl: 0.302-0.696, $P<0.001$; CM, HR: 0.305 and $95 \% \mathrm{Cl}: 0.179-0.519, P<0.001)$. In ACS patients, the LVFS was an independent predictor for ACM and CM. In stable CAD patients, the LVFS was an independent predictor only for CM. 
Previous study has shown that asymptomatic left ventricular (LV) systolic dysfunction is associated with adverse outcomes, including heart failure (HF) and all-cause mortality in the general population[14]. Cheng $S$ et al have also demonstrated that distinct components of LV mechanical function is associated with specific CVD outcomes in large, community-based sample[15]. LV dysfunction is predictive of early and late mortality after PCI[16]. LVEF, an important marker of left ventricular systolic function, is ratio of chamber volume ejected in systole (stroke volume) to the volume of the blood in the ventricular end diastolic phase (end diastolic volume)[6]. And registry data over the past two decades have identified impaired LVEF as an independent predictor of in-hospital mortality[17]. Patients with stable CAD, LVEF was independently associated with ACM (HR [for EF $\leq 35 \%$ vs. EF $\geq 60 \%$ ] 3.93, 95\% Cl 2.60-5.93; $P<0.0001)$ and with $\mathrm{CM}(\mathrm{HR} 7.12,95 \% \mathrm{Cl} 3.85-$ 13.18; $P<0.0001)$ [18]. Patients who had LVEF $<60 \%$ were 1.51 times $(95 \% \mathrm{Cl} 1.016-2.230, P=0.041)$ more likely to have MACE than those who had LVEF $\geqq 60 \%$ during follow up after undergoing successful primary PCI[19]. However LVFS, as another index of left ventricular systolic function[20], did not enter the field of vision of researchers, almost no research has focus on the relationship between LVFS and clinical outcome of CAD patients. In our retrospective cohort study, the predictive of LVFS for adverse outcomes is not inferior to LVEF( Fig. 1), low serum LVFS level was found to be independently associated ACM and CM during long term follow up, and this association remained significant after adjustments with some baseline confounders.

The mechanism of low LVFS and adverse outcome is thought to be multifactorial. Patients with LV impairment are more likely to occur sudden deaths, the risk for both sudden death and HF death is more than doubled in the lowest LVEF category[21]. The adverse outcome in the low LVFS group may relate to the increased likelihood of arrhythmia or haemodynamic decompensation[22], as well as the fact that ischaemic cardiomyopathy is a marker of more extensive and complex coronary disease, which in turn increases the risk of periprocedural complications[23]. Besides, patients with left ventricular dysfunction tended to have more severity and complexity of coronary lesions than those with normal left ventricular function[24], resulting in multivessel intervention.

There are several advantages of our study. One strength is the large sample size and the large sample may make our results more reliable. The other strength is the long-term follow-up for all included patients. Whereas, the limitations in our study should be detailed. First, because of only the baseline LVFS level collected, the dynamic change of the variable did not be analyzed. Second, this is a single retrospective cohort design. Results of our study need to be further verified by a multi-center, prospective study.

\section{Conclusion}

In conclusion, LV impairment remains a strong predictor of early and late mortality in patients with CAD even after PCI. As a consequence, assessment of LV function is integral to risk stratification and patient optimization before $\mathrm{PCl}$ and should be recommended, where feasible, before PCI. In addition to LVEF, LVFS is also a reliable and independent predictor of adverse outcomes in patients with CAD.

\section{Abbreviations}

LV: left ventricular ;LVFS:Left ventricular fractional shortening; LVEDD:LV end-diastolic dimension; LVESD:LV end-systolic dimension; LVEF:left ventricular ejection fraction; PCI:percutaneous coronary intervention; ACM:all-cause mortality; CM:cardiac mortality; MACE:major adverse cardiovascular Events; MACCE:main adverse cardiovascular and cerebrovascular events; CAD:coronary artery disease; SCAD:stable coronary artery disease; ACS:acute coronary syndrome; HDL-C:high-density lipoprotein cholesterol; HF:heart failure; DM:diabetes mellitus; ACEl:angiotensin-converting enzyme inhibitor; ARB:angiotensin II receptor blocker; CCB:calcium channel blocker; Cr:creatinine; UA:uric acid; TC:total cholesterol; LDL-C:low-density lipoprotein cholesterol; BUN:blood urea nitrogen; TG:triglyceride; SBP:systolic blood pressure; DBP:diastolic blood pressure; $\mathrm{Hb}$ hemoglobin;IVSd:interventricular septal thickness at end diastole;LVPWd:left ventricular posterior wall thickness at end diastole.

\section{Declarations}

\section{Ethics approval and consent to participate}

This study complies with the Declaration of Helsinki and the protocol was approved by the ethics committee of the First Affiliated Hospital of Zhengzhou University(Reference number:2019-ky-005). Because of the retrospective design of the study, the need to obtain informed consent from eligible patients was waived by the ethics committee.

\section{Consent for publication}

All authors are consent for publication.

\section{Availability of data and material}


Due to confidentiality policies, data will not be shared.

\section{Competing interests}

The authors declare that they have no competing interests.

\section{Funding}

This research was funded by the National Natural Science Foundation of China $(81870328,81760043$ and 81800267$)$ and China Postdoctoral Science Foundation (2019M652593). The funding institutions had no role in the design of the study, collection, analysis and interpretation of data, and in writing the manuscript.

\section{Authors' contributions}

JY Zhang and YY Zheng conceived and designed the study; QQ Guo and YY Zheng contributed to the design; QQ Guo performed the study; JN Tang , XM Yang, ZL Zhang, JC Zhang, MD Cheng, FH Song, ZY Liu, K Wang, LZ Jiang, L Fan, XT Yue, Y Bai, XY Dai and RJ Zheng help to the study;QQ Guo and YY Zheng analyzed the data and draft the paper; JN Tang contributed to the drafting. All authors read,critically revised the final manuscript. All authors read and approved the final manuscript.

\section{Acknowledgements}

Not applicable.

\section{References}

1. Roth GA, Forouzanfar MH, Moran AE, Barber R, Nguyen G, Feigin VL, et al. Demographic and epidemiologic drivers of global cardiovascular mortality. N Engl J Med. 2015;372(14):1333-41.

2. Hong YJ, Jeong MH, Lee SH, Park OY, Jeong WK, Lee SR, et al. The long-term clinical outcomes after rescue percutaneous coronary intervention in patients with acute myocardial infarction. J Interv Cardiol. 2003;16(3):209-16.

3. Tian L, Zhu J, Liu L, Liang Y, Li J, Yang Y. Hemoglobin A1c and short-term outcomes in patients with acute myocardial infarction undergoing primary angioplasty: an observational multicenter study. Coron Artery Dis. 2013;24(1):16-22.

4. Sahn DJ, DeMaria A, Kisslo J, Weyman A. Recommendations regarding quantitation in M-mode echocardiography: results of a survey of echocardiographic measurements. Circulation. 1978;58(6):1072-83.

5. Devereux RB, Reichek N. Echocardiographic determination of left ventricular mass in man. Anatomic validation of the method. Circulation. 1977;55(4):613-8.

6. Kosaraju A, Goyal A, Grigorova Y, Makaryus AN. (2020). Left Ventricular Ejection Fraction. In StatPearls. StatPearls Publishing.

7. Moon YJ, Kim JW, Bang YS, Lim YS, Ki Y, Sang BH. Prediction of all-cause mortality after liver transplantation using left ventricular systolic and diastolic function assessment. PLoS One. 2019;14(1):e0209100.

8. Bonhorst D, Guerreiro S, Fonseca C, Cardim N, Macedo F, Adragão P. Real-life data on heart failure before and after implantation of resynchronization and/or defibrillation devices - the Sincrone study. Rev Port Cardiol. 2019;38(1):33-41.

9. Song L, Brezden-Masley C, Ramanan V, Ghugre N, Barfett JJ, Chan K, et al. Serial Measurements of Left Ventricular Systolic and Diastolic Function by Cardiac Magnetic Resonance Imaging in Patients with Early Stage Breast Cancer on Trastuzumab. Am J Cardiol. 2019;123(7):1173-9.

10. Aronow WS, Fleg JL, Pepine CJ, Artinian NT, Bakris G, Brown AS, et al. ACCF/AHA 2011 expert consensus document on hypertension in the elderly: a report of the American College of Cardiology Foundation Task Force on Clinical Expert Consensus documents developed in collaboration with the American Academy of Neurology, American Geriatrics Society, American Society for Preventive Cardiology, American Society of Hypertension, American Society of Nephrology, Association of Black Cardiologists, and European Society of Hypertension. J Am Coll Cardiol. 2011;57(20):2037-114.

11. Olafsdottir E, Andersson DK, Dedorsson I, Stefánsson E. The prevalence of retinopathy in subjects with and without type 2 diabetes mellitus. Acta Ophthalmol. 2014;92(2):133-7.

12. Hicks KA, Tcheng JE, Bozkurt B, Chaitman BR, Cutlip DE, Farb A, et al., 2014 ACC/AHA Key Data Elements and Definitions for Cardiovascular Endpoint Events in Clinical Trials: A Report of the American College of Cardiology/American Heart Association Task Force on Clinical Data Standards (Writing Committee to Develop Cardiovascular Endpoints Data Standards). J Am Coll Cardiol, 2015. 66(4): p. 403-469. 
13. Hicks KA, Mahaffey KW, Mehran R, Nissen SE, Wiviott SD, Dunn B, et al. 2017 Cardiovascular and Stroke Endpoint Definitions for Clinical Trials. Circulation. 2018;137(9):961-72.

14. Wang TJ, Evans JC, Benjamin EJ, Levy D, LeRoy EC, Vasan RS. Natural history of asymptomatic left ventricular systolic dysfunction in the community. Circulation. 2003;108(8):977-82.

15. Cheng S, McCabe EL, Larson MG, Merz AA, Osypiuk E, Lehman BT, et al. Distinct Aspects of Left Ventricular Mechanical Function Are Differentially Associated With Cardiovascular Outcomes and All-Cause Mortality in the Community. J Am Heart Assoc.

2015;4(10):e002071.

16. De Silva K, Webb I, Sicard P, Lockie T, Pattinson S, Redwood S, et al. Does left ventricular function continue to influence mortality following contemporary percutaneous coronary intervention? Coron Artery Dis. 2012;23(3):155-61.

17. Grayson AD, Moore RK, Jackson M, Rathore S, Sastry S, Gray TP, et al. Multivariate prediction of major adverse cardiac events after 9914 percutaneous coronary interventions in the north west of England. Heart. 2006;92(5):658-63.

18. Lamblin N, Meurice T, Tricot O, Lemesle G, Deneve M, de Groote P, et al. Effect of left ventricular systolic dysfunction on secondary medical prevention and clinical outcome in stable coronary artery disease patients. Arch Cardiovasc Dis. 2017;110(1):35-41.

19. Son YJ, Shim SK, Hwang SY, Ahn JH, Yu HY. Impact of left ventricular ejection fraction and medication adherence on major adverse cardiac events during the first year after successful primary percutaneous coronary interventions. J Clin Nurs. 2016;25(7-8):1101-11.

20. Recommendations for Cardiac Chamber Quantification by Echocardiography in Adults. An Update from the American Society of Echocardiography and the European Association of, Cardiovascular Imaging. Eur Heart J Cardiovasc Imaging. $2016 ; 17(4): 412$.

21. Hall TS, von Lueder TG, Zannad F, Rossignol P, Duarte K, Chouihed T, et al. Relationship between left ventricular ejection fraction and mortality after myocardial infarction complicated by heart failure or left ventricular dysfunction. Int J Cardiol. 2018;272:260-6.

22. Perera D, Stables R, Thomas M, Booth J, Pitt M, Blackman D, et al. Elective intra-aortic balloon counterpulsation during high-risk percutaneous coronary intervention: a randomized controlled trial. JAMA. 2010;304(8):867-74.

23. Januszek R, Dziewierz A, Siudak Z, Rakowski T, Dudek D, Bartuś S, et al. Chronic obstructive pulmonary disease and periprocedural complications in patients undergoing percutaneous coronary interventions. PLoS One. 2018;13(10):e0204257.

24. Ferreira JP, Rossignol P, Demissei B, Sharma A, Girerd N, Anker SD, et al. Coronary angiography in worsening heart failure: determinants, findings and prognostic implications. Heart. 2018;104(7):606-13.

\section{Figures}




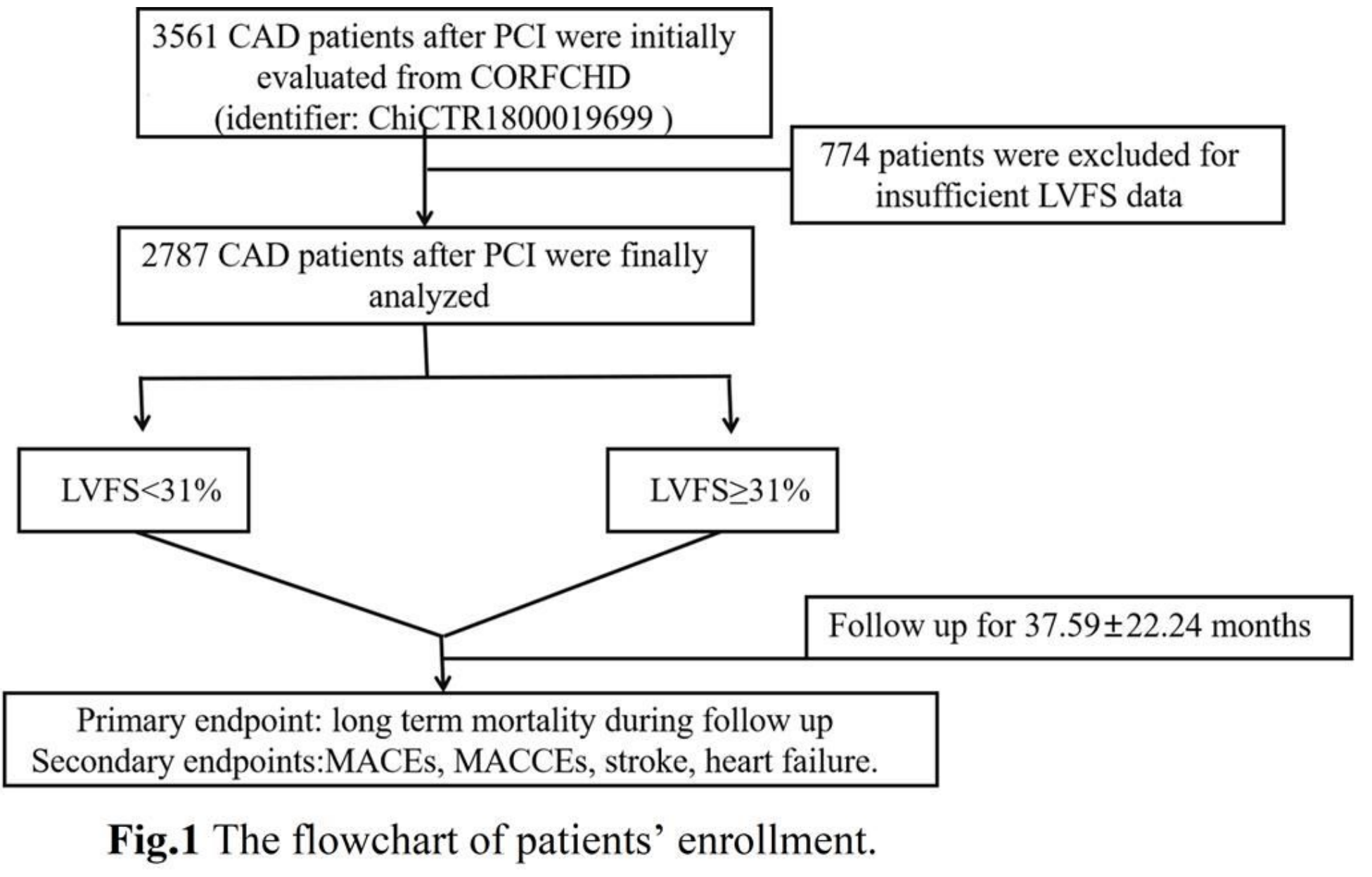

Figure 1

The flowchart of patients' enrollment. 


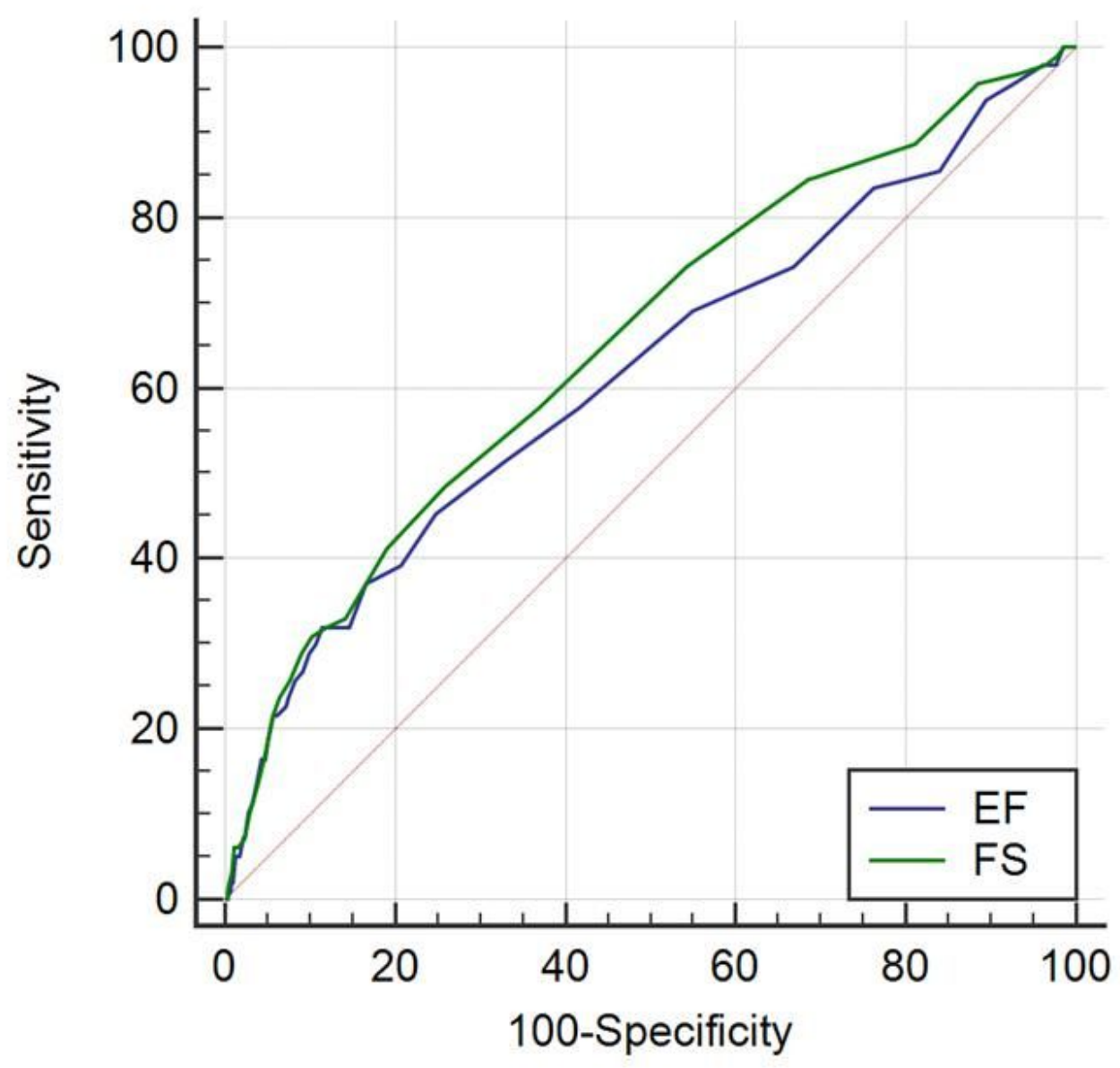

Figure 2

Receiver-operating characteristic analysis of EF and FS in prediction of adverse outcomes in CAD patients. 

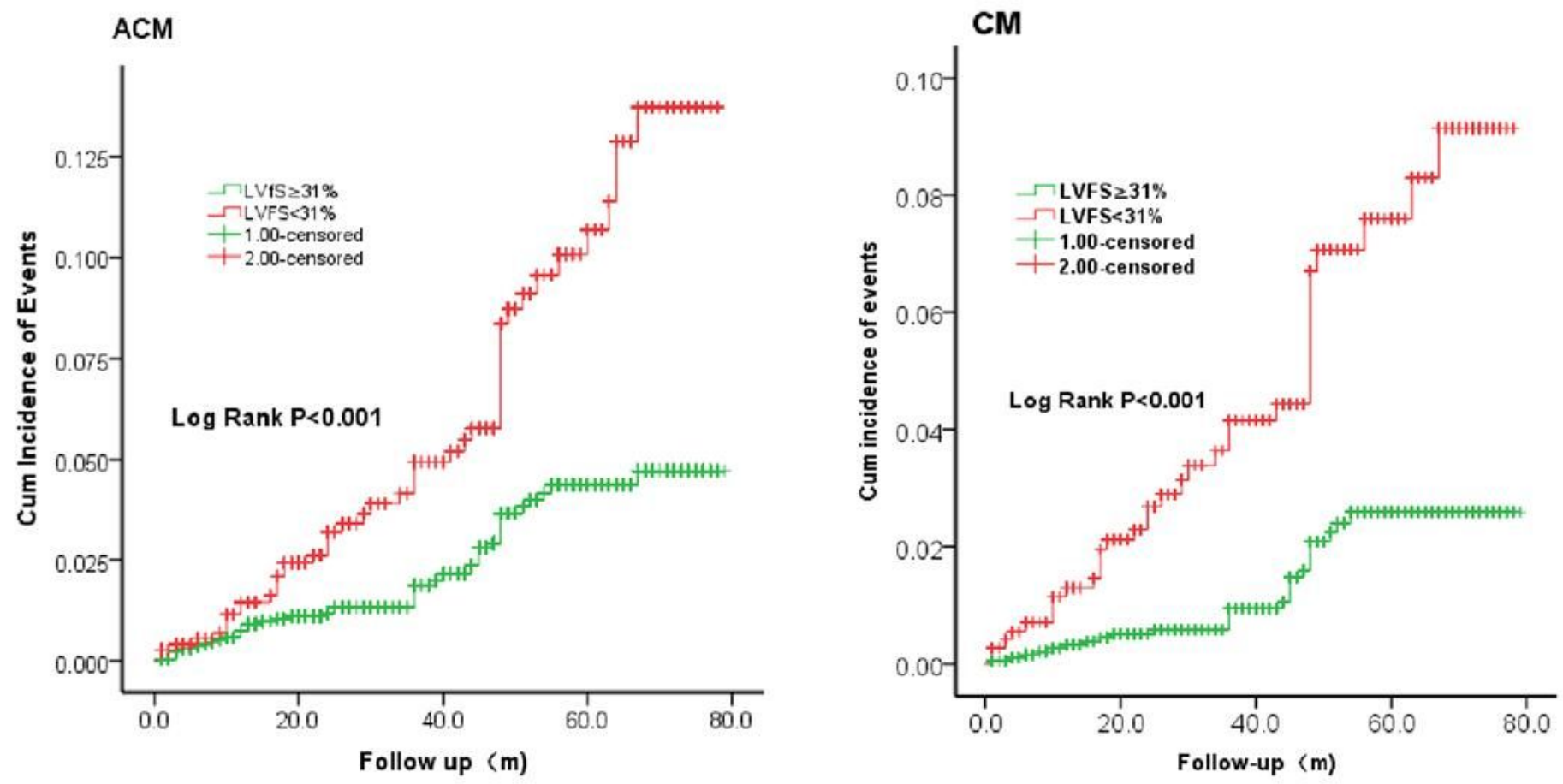

Fig. 3 Cumulative Kaplan-Meier estimates of the time to the first adjudicated occurrence of $\mathrm{ACM}$ and $\mathrm{CM}$ in total CAD patients.

Figure 3

Cumulative Kaplan-Meier estimates of the time to the first adjudicated occurrence of ACM and CM in total CAD patients.
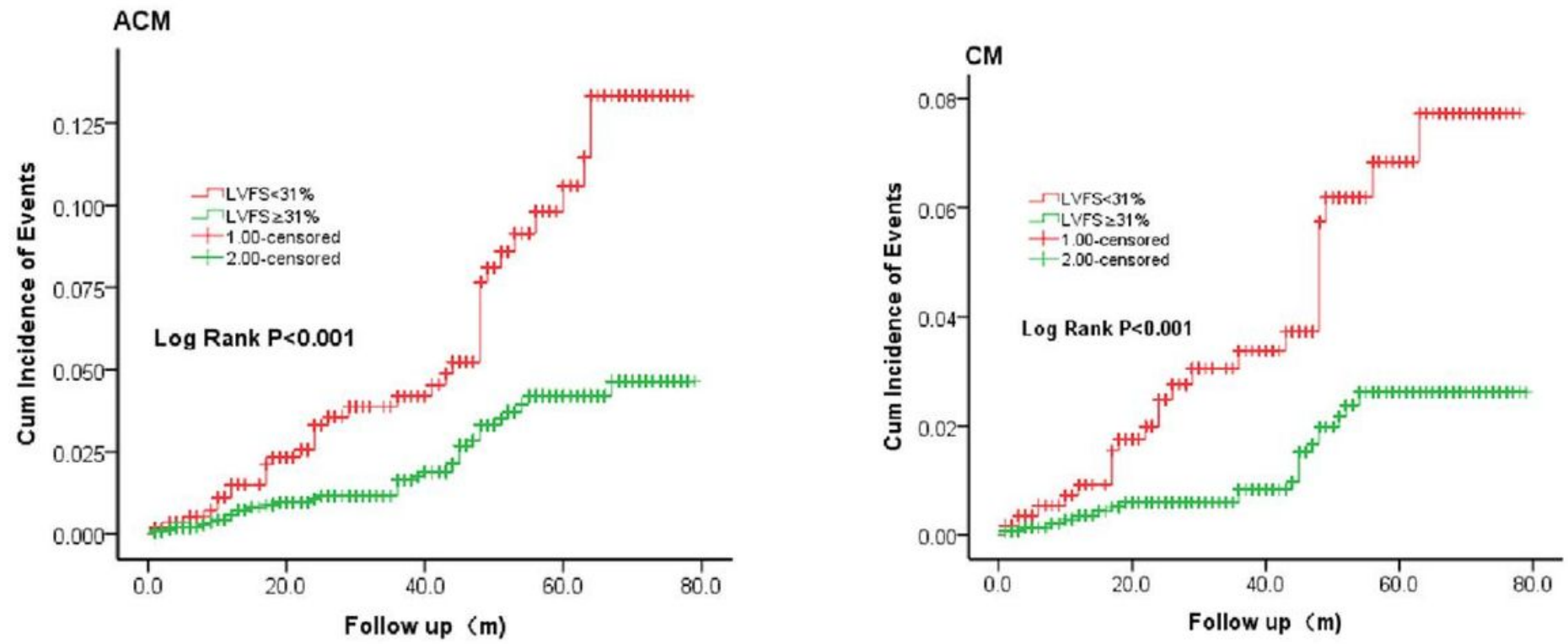

Fig. 4 Cumulative Kaplan-Meier estimates of the time to the first adjudicated occurrence of $\mathrm{ACM}$ and $\mathrm{CM}$ in ACS patients.

Figure 4 

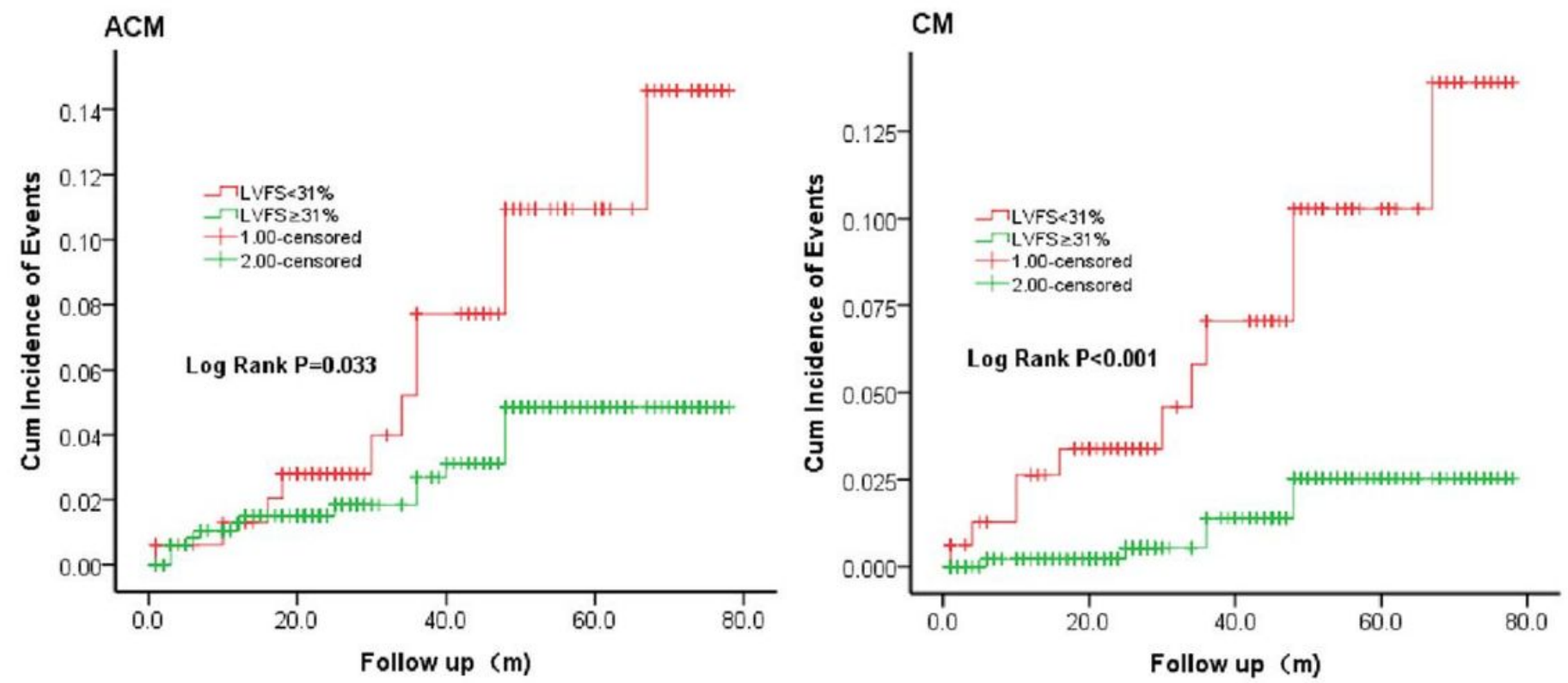

Fig. 5 Cumulative Kaplan-Meier estimates of the time to the first adjudicated occurrence of $\mathrm{ACM}$ and $\mathrm{CM}$ in SCAD patients.

Figure 5

Cumulative Kaplan-Meier estimates of the time to the first adjudicated occurrence of ACM and CM in SCAD patients. 